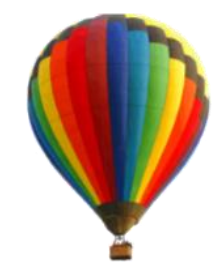

\title{
MACROFAUNA BENTÔNICA SOB EFEITO DA PISCICULTURA DE TANQUE- REDE NO RIO SÃO MATEUS, SUDESTE DO BRASIL
}

\author{
BENTHIC MACROFAUNA UNDER THE EFFECT OF THE CAGE FISH CULTURE AT \\ SÃO MATEUS RIVER, SOUTHEAST OF BRAZIL
}

\section{Jociane Liandro Rezeno1*, Nálita Maria Scamparle Teodoro2, \& Karla Gonçalves da Costa3 \\ 13 Núcleo de Bentos Marinho do Departamento de Ciências Agrárias e Biológicas do Centro Universitário Norte do Espírito Santo da Universidade Federal do Espírito Santo. 2 Laboratório de Biodiversidade Bentônica Marinha do Departamento de Biologia Animal do Instituto de Biologia da Universidade Estadual de Campinas. 1*jocianerezeno@gmail.com 2 nalita.scamparle@gmail.com 3 karlacostabio@gmail.com}

\section{ARTIGO INFO.}

Recebido em: 01.07.2020

Aprovado em: 31.07.2020

Disponibilizado em: 15.09 .2020

\section{Palavras-chave:}

Qualidade ambiental; impacto antrópico, BMWP'; enriquecimento orgânico.

\section{KEYWORDS:}

Environmental quality; anthropic impact; BMWP'; organic enrichment.

\section{*Autor Correspondente: Rezeno, J. L.}

\section{RESUMO}

A piscicultura no Brasil vem crescendo em ritmo acelerado e isto se deve ao alto grau de lucratividade e retorno de investimento rápido. Entretanto, a escolha de local não favorável para a implantação de tanquesrede pode resultar na produção de efluente de baixa qualidade, baixa concentração de oxigênio dissolvido, altas concentrações de matéria orgânica, nutrientes e sólidos em suspensão, os quais afetam os ambientes aquáticos. Dessa forma, o monitoramento da comunidade bentônica pode ser usado como uma ferramenta de avaliação ambiental, refletindo mudanças nas condições físicas, químicas e ecológicas do local. Assim, o objetivo deste estudo foi avaliar a comunidade macrobentônica sob tanques rede em um trecho do rio São Mateus, ES, a fim de detectar possíveis alterações decorrentes dessa atividade. As amostras foram coletadas cinco vezes entre fevereiro de 2011 e maio de 2012, em quatro pontos de amostragem: sob o tanque de peixes, na margem oposta, cerca de $500 \mathrm{~m}$ a montante e $500 \mathrm{~m}$ a jusante. As amostras foram coletadas com um coletor Van Veen, quantificadas e identificadas em laboratório. Foram encontrados 195 indivíduos distribuídos em cinco táxons, com Oligochaeta e Chironomidae sendo os mais abundantes. Apenas variações temporais foram detectadas. $\mathrm{O}$ índice BMWP' deixa claro que a qualidade ambiental de toda a área de estudo é muito crítica, demonstrando que a degradação ambiental do rio causada por diversos fatores, incluindo a descarga de efluentes orgânicos, parece comprometer muito mais a fauna bentônica do local do que a própria piscicultura.

\begin{abstract}
The rapid growth of fish farming in Brazil is due to the high degree of profitability and rapid return on investment. However, the choice of an unfavourable location for the implementation of network tanks can result in the production of low-quality effluents, low concentration of dissolved oxygen, high concentrations of organic matter, nutrients and suspended solids, which affect aquatic environments. In this way, the monitoring of the benthic community can be used as an environmental assessment tool, reflecting changes in the physical, chemical and ecological conditions of the place. Thus, the objective of this study was to evaluate the macrobenthic community under net cage in a stretch of the São Mateus river, ES, in order to detect possible changes resulting from this activity. Five samplings were carried out between February 2011 and May 2012, at four sampling points: under the fish tank, on the opposite marge, $500 \mathrm{~m}$ upstream and $500 \mathrm{~m}$ downstream. The samples were collected with a Van Veen grab and the macrofauna was quantified and identified in the laboratory. 195 individuals were found and Oligochaeta and Chironomidae were most abundant. Only temporal variations were detected. The BMWP index showed that the environmental quality of the entire study area is very critical, demonstrating that the environmental degradation of the river caused by several factors, including the release of organic effluents, seems to compromise the local benthic fauna much more than the fish farming itself.
\end{abstract}


Citação (APA): Rezeno, J. L., Costa, K. G., \& Teodoro, N. M. S. (2020). Macrofauna bentônica sob efeito da piscicultura de tanque-rede no rio São Mateus, sudeste do Brasil. Brazilian Journal of Production Engineering, 6(8), Edição Especial "Pesca e Aquicultura: Gestão, Política e Inovação", 01-13.

\section{INTRODUÇÃO}

A piscicultura em tanques-rede é uma prática de origem asiática que pode ser implantada nos mais diferentes corpos d'água, permitindo o cultivo de diferentes espécies, além de assegurar maior controle do estoque e observação dos peixes (Barbosa, 2010). No Brasil, esta atividade cresce em ritmo acelerado e isto se deve ao alto grau de lucratividade e rápido retorno de capital (Martins, 2016).

O cultivo da tilápia, principal peixe criado em tanques-redes no país, chegou a 300 mil toneladas em 2017 (Barroso, Muñoz, \& Cai, 2019). Entretanto, o manejo inadequado e a escolha de local não favorável para a implantação de tanques-rede podem impactar negativamente o meio ambiente, causando aumento das concentrações de matéria orgânica, eutrofização, redução do teor de oxigênio da água e consequentemente da produtividade influenciando a viabilidade econômica (Vieira, 2018). A seleção do local para o cultivo de peixes é um fator-chave na aquicultura, afetando tanto o sucesso quanto a sustentabilidade (Óscar, Lindsay, Trevor, \& Barquin, 2003).

A instalação de tanques-rede pode interferir na dinâmica dos ecossistemas costeiros e oceânicos. O principal problema envolvendo este tipo de cultivo é a grande quantidade de dejetos orgânicos lançados diretamente no ambiente, principalmente fezes e restos de ração. Estes resíduos chegam no ambiente aquático na forma de nitrogênio, fósforo e material particulado que pode contribuir para o processo de eutrofização artificial, comprometendo a produção primária, influenciando na dinâmica do oxigênio dissolvido e interferindo no desenvolvimento dos peixes (Huang, Huang, Hsieh, Meng, \& Chen, 2012; Cardoso, El-Deir, \& Cunha, 2016; Moura, Lopes, \& Henry-Silva, 2014).

Dessa forma, a comunidade bentônica desempenha um importante papel nos processos ecológicos e ecotoxicológicos, refletindo as alterações físicas, químicas e ecológicas que ocorrem no ambiente (Griffiths, Kadin, Nascimento, Tamelander, Törnross, Bonaglia, Bonsdorff et al., 2017). Estes organismos funcionam como indicadores de alterações da qualidade ambiental, indicando o estado trófico e os efeitos de poluentes e de descargas orgânicas nos corpos d'água (Menezes \& Beyruth, 2003). Por exemplo, larvas de insetos Chironomidae e de anelídeos Oligochaeta resistem a baixas concentrações de oxigênio dissolvido e, portanto, são comuns em ambientes eutróficos (Pastorino, Pizzul, Bertoli, Perilli, Brizio, \& Salvi, 2019). Já em ambientes aquáticos de águas limpas e bem oxigenadas, com baixa quantidade de nutrientes, a abundância de insetos das ordens Ephemeroptera, Plecoptera e Trichoptera é frequente, por serem organismos sensíveis à poluição, podendo ser indicadores de ambientes oligotróficos (Ceneviva-Bastos, Prates, Romero, Bispo, \& Casatti, 2017).

Com bases nestas informações e partindo da hipótese que a macrofauna bentônica seja afetada pelo cultivo de peixes em tanques-redes, propusemos um estudo investigando essa situação em um rio do sudeste do Brasil.

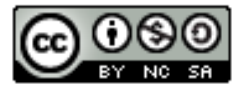


Citação (APA): Rezeno, J. L., Costa, K. G., \& Teodoro, N. M. S. (2020). Macrofauna bentônica sob efeito da piscicultura de tanque-rede no rio São Mateus, sudeste do Brasil. Brazilian Journal of Production Engineering, 6(8), Edição Especial "Pesca e Aquicultura: Gestão, Política e Inovação", 01-13.

\section{MATERIAL E MÉTODOS}

\section{Área de Estudo}

O estudo foi desenvolvido em um trecho do rio São Mateus, norte do Espírito Santo, sudeste do Brasil. O cultivo de peixes foi implantado nesse local devido a carência econômica, social e tecnológica da comunidade e a parceria formada na época entre o Centro Universitário Norte do Espírito Santo (CEUNES), da Universidade Federal do Espírito Santo (UFES), com a APESAM (Associação de Pescadores de São Mateus) (Paiva, Casotti, Rodrigues, \& Freitas, 2015).

O município de São Mateus tem topografia predominantemente plana à suavemente ondulada e o clima é seco subúmido com influência do vento Nordeste. O regime de chuvas influencia diretamente na agricultura local, dinâmica florestal, e na manutenção de grandes córregos e rios, dentre os quais, destaca-se o Rio Itaúnas e o Rio Cricaré, que nasce em Minas Gerais. O índice de precipitação pluviométrica anual é de $1.313 \mathrm{~mm}$, com chuvas mais intensas distribuídas entre os meses de outubro e abril (PROATER, 2011).

As coletas de macrofauna bentônica foram realizadas às margens do rio São Mateus onde estavam os tanques-rede, próximo a comunidade de pescadores tradicionais de Pedra d'Água (18 $43^{\circ} 05.86^{\prime \prime} \mathrm{S}$ e $39^{\circ} 48^{\prime} 50.38^{\prime}$ ' O). Este rio é a principal fonte de abastecimento para várias povoações e cidades e fornece água para inúmeras áreas de irrigação, sendo também receptor de efluentes domésticos e industriais (Figura 1). O monitoramento dos tanques era feito de três em três dias, para identificar possíveis danos e, caso sejam encontrados, consertá-los (Assis \& Freitas, 2012). De acordo com estudos anteriores, as áreas onde estão localizadas a piscicultura e o lançamento de efluentes domésticos apresentaram condições ambientais não favoráveis para práticas aquícolas (Almeida, 2012; Mamão, 2012; Pereira, 2012; Rezeno, 2012).

Figura 1. Área de estudo, com a marcação dos quatro pontos de amostragem, localizados no Rio São Mateus, Espírito Santo, sudeste do Brasil.
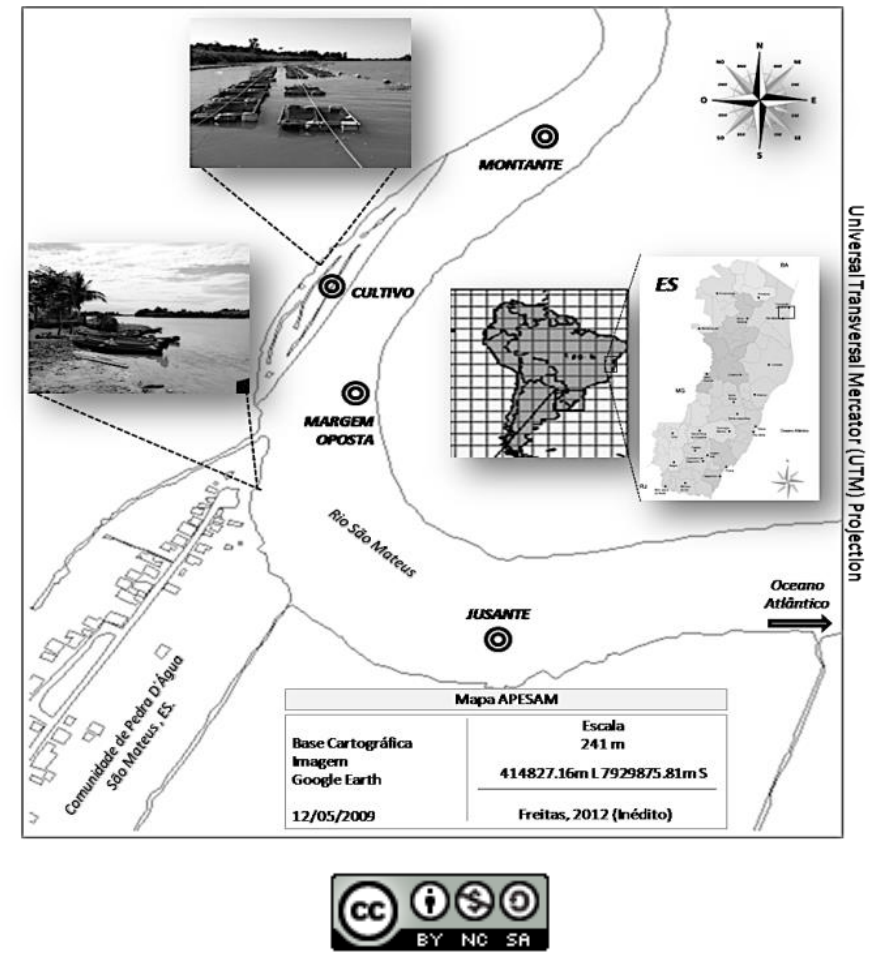
Citação (APA): Rezeno, J. L., Costa, K. G., \& Teodoro, N. M. S. (2020). Macrofauna bentônica sob efeito da piscicultura de tanque-rede no rio São Mateus, sudeste do Brasil. Brazilian Journal of Production Engineering, 6(8), Edição Especial "Pesca e Aquicultura: Gestão, Política e Inovação", 01-13.

O cultivo começou em 2003, com exclusividade para a tilápia (Oreochromis niloticus) até 2008, quando foi introduzido também o cultivo de robalo-peva (Centropomus parallelus). $\mathrm{O}$ arraçoamento era realizado três vezes ao dia, sendo lançados, ao todo, $600 \mathrm{~kg}$ de ração, distribuídos pelos 70 tanques-rede, sendo 13 deles de robalos. Cada tanque-rede possuía 4m2, comportando 1000 indivíduos de tilápia, quando alevinos, e 500 indivíduos, quando adultos. Os tanques de robalo comportavam 180 alevinos e 350 adultos. A despesca da tilápia era realizada a cada seis meses, enquanto do robalo ocorria a cada 18 meses, aproximadamente. Segundo Paiva et al. (2015) todo o descarte dos rejeitos do pescado é realizado diretamente nas margens do rio São Mateus.

\section{Procedimentos de Campo}

Entre fevereiro de 2011 e maio de 2012, foram realizadas cinco amostragens em quatro pontos de amostragem (Figura 1): 1- sob o cultivo, 2- na margem oposta, 3- cerca de 500m à montante e 4- 500m à jusante, com o auxílio de um pegador de sedimento do tipo Van Veen $(0,08 \mathrm{~m} 2)$. A primeira amostragem (fev/11) ocorreu num momento que havia apenas tilápias no local e antecedeu a introdução de alevinos de robalos, que ocorreu em abr/11. A segunda amostragem (ago/11) ocorreu quatro meses após a introdução de robalos, a terceira amostragem ocorreu em dezembro de 2011, a quarta em abril de 2012 e a quinta amostragem (mai/12) ocorreu após a troca dos alevinos e introdução de nova fórmula de ração.

As amostras foram coletadas sempre em triplicatas. $\mathrm{O}$ volume do sedimento coletado para as análises da macrofauna bentônica foi padronizado em um pote com volume de $0,002 \mathrm{~m} 3$, lavado no próprio local em malha de $300 \mu \mathrm{m}$ e acondicionado em sacos plásticos devidamente etiquetados com formaldeído a 4\%. Uma amostra de sedimento foi retirada para análise de granulometria e matéria orgânica em cada ponto amostral. Estas foram acondicionadas em sacos plásticos devidamente etiquetados e levados ao laboratório. Por problemas operacionais, na coleta de abril/12 não foi possível coletar o sedimento no ponto Cultivo.

\section{Procedimentos de Laboratório}

Em laboratório, as amostras para análises de sedimento foram submetidas à secagem na estufa a $60^{\circ} \mathrm{C}$. Teores de matéria orgânica do sedimento foram determinados através de combustão em mufla à $550^{\circ} \mathrm{C}$ por 1 hora. A granulometria do sedimento foi determinada por rotinas de peneiramento (Suguio, 1973).

As amostras para análise da comunidade bentônica foram triadas em microscópio estereoscópico e os organismos encontrados foram armazenados em álcool a $70 \%$. Posteriormente, os organismos foram quantificados e identificados sob microscópio estereoscópico e ótico com o auxílio de bibliografia adequada.

\section{Tratamento Estatístico}

Para testar se o cultivo de peixes em tanques-redes influencia a estrutura da comunidade macrobentônica foram realizadas análises univariadas e multivariadas. Como parâmetros univariados da fauna foram utilizados os valores de abundância, riqueza (número de táxons) e diversidade de Shannon-Wiener $\left(H^{\prime}, \log _{2}\right)$. Para testar a significância dos descritores 
Citação (APA): Rezeno, J. L., Costa, K. G., \& Teodoro, N. M. S. (2020). Macrofauna bentônica sob efeito da piscicultura de tanque-rede no rio São Mateus, sudeste do Brasil. Brazilian Journal of Production Engineering, 6(8), Edição Especial "Pesca e Aquicultura: Gestão, Política e Inovação", 01-13.

univariados da fauna e os fatores abióticos entre os meses de coletas e pontos amostrados foram aplicadas análises de variância bifatorial (ANOVA) $(p=0,05)$. Testes de Cochran foram aplicados para verificar a homogeneidade das variâncias e os dados foram transformados em $\log (\mathrm{x}+1)$ quando necessário. Matrizes de similaridade foram construídas utilizando o índice de similaridade de Bray-Curtis a partir dos dados da macrofauna bentônica (sem transformação).

Os dados foram ordenados e plotados através da análise de proximidade (MDS, Multidimensional Scaling) e a diferença dos dados da fauna entre os meses e os pontos foram testadas através da análise não paramétrica ANOSIM (Clarke \& Green, 1988). A análise BIOENV foi utilizada para determinar as possíveis relações entre a estrutura multivariada da comunidade e combinações de variáveis ambientais (Clarke, \& Ainsworth 1993). Para a realização das análises estatísticas foram utilizados os aplicativos STATISTICA® v.7 e PRIMER ${ }_{\circledast}$ v.6 e para o processamento dos dados das análises granulométricas o SysGran $\circledast$ v.3.0.

Além disso, foi utilizado o índice biótico BMWP', uma adaptação feita por Alba-Tercedor (1996) do índice original BMWP (Biological Monitoring Working Party). O índice BMWP baseia-se na soma de valores de tolerância (sensibilidade a poluentes orgânicos) atribuídos a cada grupo de macroinvertebrado de acordo com sua capacidade de sobreviver em diferentes situações de qualidade de água (Tabela 1). Este índice é qualitativo, levando em conta a presença ou ausência de famílias. Para interpretar as pontuações totais do índice BMWP', foi utilizada a tabela de valores correlacionados a cinco graus diferentes de contaminação da água e suas respectivas cores (Tabela 2), propostas por Alba-Tercedor (1996).

Tabela 1. Valores de tolerância a poluentes atribuídos aos táxons (Alba-Tercedor, 1996).

$$
\text { FAMÍLIAS }
$$

Siphlonuridae, Heptageniidae, Leptophlebiidae, Potamanthidae, Taeniopterygidae, Leuctridae, Capniidae, Perlodidae, Perlidae, Chloroperlidae, Aphelocheiridae, Phryganeidae, Molannidae, Beareidae, Odontoceridae, Leptoceridae, Goeridae, Lepidostomatidae, Brachycentridae, Sericostomatidae, Calamoceratidae, Helicopsychidae, Megapodagrionidae, Atheridae, Blephariceridae

Astacidae, Lestidae, Calopterygidae, Gomphidae, Cordulegastridae, Aeshnidae, Corduliidae, Libellulidae, Psychomyiidae, Philopotamidae, Glossosomatidae

Ephemerellidae, Prosopistomatidae, Nemouridae, Gripopterygidae, Rhyacophilidae, Polycentropodidae, Limnephilidae, Ecnomidae, Hydrobiosidae, Pyralidae, Psephenidae

Neritidae, Viviparidae, Ancylidae, Thiaridae, Hydroptilidae, Unionidae, Mycetopodidae, Hyriidae, Corophilidae, Gammaridae, Hyalellidae, Atylidae, Palaemonidae, Trichodactylidae, Platycnemis, Coenagrionidae, Leptohyphidae

Oligoneuridae, Polymitarciydae, Dryopidae, Elmidae, Helophoridae, Hydrochidae, Hydraenidae, Clambidae, Hydropsychidae, Tipulidae, Simulidae, Planariidae, Dendrocoelidae, Dugesiidae

Aeglidae, Baetidae, Caenidae, Haliplidae, Curculionidae, Chrysomelidae, Tabanidae, Stratiomyidae, Empididae, Dolichopodidae, Dixidae, Ceratopogonidae, Anthomyiidae, Limoniidae, Psychodidae, Sciomyzidae, Rhagionidae, Sialidae, Corydalidae, Piscicolidae, Hydracarina

Mesoveliidae, Hydrometridae, Gerridae, Nepidae, Naucoridae (Limnocoridae), Pleidae, Notonectidae, Corixidae, Veliidae, Helodidae, Hygrobiidae, Lymnaeidae, Physidae, Planorbidae, Bithynellidae, Sphaeriidae, Glossiphoniidae, Hirudinea, Erpobdellidae, Asellidae, Ostracoda

\section{Fonte: Autores}


Citação (APA): Rezeno, J. L., Costa, K. G., \& Teodoro, N. M. S. (2020). Macrofauna bentônica sob efeito da piscicultura de tanque-rede no rio São Mateus, sudeste do Brasil. Brazilian Journal of Production Engineering, 6(8), Edição Especial "Pesca e Aquicultura: Gestão, Política e Inovação", 01-13.

Tabela 2. Correlação das pontuações totais dos organismos encontrados em um determinado ponto de coleta e as cores correspondentes à qualidade da água (Alba-Tercedor, 1996).

\begin{tabular}{|c|c|c|c|c|}
\hline CLASSE & QUANTIDADE & VALOR & SIGNIFICADO & COR \\
\hline I & $\mathrm{BOA}$ & $101-120>120$ & $\begin{array}{l}\text { Águas muito limpas (águas } \\
\text { prístinas) } \\
\text { Águas não poluídas ou sistema } \\
\text { perceptivelmente não alterado }\end{array}$ & Azul \\
\hline II & ACEITÁVEL & $61-100$ & $\begin{array}{l}\text { São evidentes efeitos moderados de } \\
\text { poluição }\end{array}$ & Verde \\
\hline III & DUVIDOSA & $36-60$ & Águas poluídas (sistema alterado) & Amarelo \\
\hline IV & CRÍTICA & $16-35$ & $\begin{array}{l}\text { Águas muito poluídas (sistema } \\
\text { muito alterado) }\end{array}$ & Laranja \\
\hline $\mathrm{V}$ & MUITO CRÍTICA & $<16$ & $\begin{array}{l}\text { Águas fortemente poluídas (sistema } \\
\text { fortemente alterado) }\end{array}$ & Vermelho \\
\hline
\end{tabular}

Fonte: Autores

\section{RESULTADOS}

\section{Variáveis ambientais}

O sedimento foi caracterizado como areia fina, pobremente selecionada. A matéria orgânica registrada variou entre $5,76 \%$ e $26,54 \%$ ao longo do estudo, com valor médio igual a $17,21 \%$, não apresentando diferenças significativas entre os pontos e entre as coletas $(p>0,05)$ (Tabelas 3 e 4$)$.

Diferenças significativas para seleção do grão, frações de areia e finos foram observadas entre os pontos de coleta. $O$ ponto Jusante apresentou maior valor significativo para a seleção do grão do que o ponto Montante. Enquanto que no ponto Cultivo foram registradas as maiores porcentagens médias de areia e as menores de silte do que os pontos Jusante e Margem Oposta (Tabelas 3 e 4).

Tabela 3. Descritores abióticos dos sedimentos amostrados próximos a piscicultura no Rio

São Mateus.

\begin{tabular}{|c|c|c|c|c|c|}
\hline & & CULTIVO & OPOSTA & MONTANTE & JUSANTE \\
\hline \multirow{4}{*}{$\begin{array}{l}\text { \% Mat. } \\
\text { Orgânica }\end{array}$} & fev/11 & 23,38 & 14,31 & 10,50 & 17,92 \\
\hline & ago/11 & 8,10 & 13,90 & 26,54 & 22,66 \\
\hline & $\mathrm{dez} / 11$ & 16,93 & 16,26 & 26,54 & 25,72 \\
\hline & abr/12 & 5,76 & 12,05 & 15,49 & 20,36 \\
\hline \multirow{4}{*}{ Seleção } & fev/11 & 1,205 & 0,971 & 0,9563 & 1,291 \\
\hline & ago/11 & 1,29 & 1,017 & 1,174 & 1,157 \\
\hline & dez/11 & 1,077 & 1,098 & 1,07 & 1,415 \\
\hline & abr/12 & 0,8419 & 1,051 & 1,075 & 1,216 \\
\hline \multirow{4}{*}{ Média } & fev/11 & 2,276 & 3,011 & 2,975 & 2,459 \\
\hline & ago/11 & 2,519 & 2,754 & 2,447 & 2,673 \\
\hline & $\mathrm{dez} / 11$ & 2,744 & 2,792 & 2,605 & 2,412 \\
\hline & abr/12 & 2,828 & 2,833 & 2,707 & 2,601 \\
\hline \multirow{3}{*}{$\%$ Finos } & fev/11 & 11,17 & 17,72 & 15,32 & 14,62 \\
\hline & ago/11 & 3,838 & 12,82 & 10,39 & 14,83 \\
\hline & $\operatorname{dez} / 11$ & 14,02 & 17,06 & 11,64 & 15,79 \\
\hline
\end{tabular}

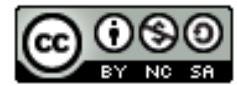


Citação (APA): Rezeno, J. L., Costa, K. G., \& Teodoro, N. M. S. (2020). Macrofauna bentônica sob efeito da piscicultura de tanque-rede no rio São Mateus, sudeste do Brasil. Brazilian Journal of Production Engineering, 6(8), Edição Especial "Pesca e Aquicultura: Gestão, Política e Inovação", 01-13.

\begin{tabular}{cccccc} 
& abr/12 & 4,107 & 16,78 & 14,11 & 17,75 \\
& fev/11 & 88,76 & 82,28 & 84,68 & 85,18 \\
\multirow{2}{*}{ \% Areia } & ago/11 & 93,71 & 87,16 & 89,00 & 85,05 \\
& dez/11 & 85,98 & 82,81 & 88,36 & 84,12 \\
& abr/12 & 95,73 & 83,17 & 85,89 & 82,01 \\
\multirow{2}{*}{ \% Cascalho } & $\mathbf{f e v / 1 1}$ & 0,07 & 0,00 & 0,00 & 0,20 \\
& $\mathbf{a g o / 1 1}$ & 2,46 & 0,03 & 0,61 & 0,12 \\
& $\mathbf{d e z} / \mathbf{1 1}$ & 0,00 & 0,13 & 0,00 & 0,10 \\
& $\mathbf{a b r} / \mathbf{1 2}$ & 0,17 & 0,05 & 0,00 & 0,24 \\
\hline
\end{tabular}

Tabela 4. Resultado da ANOVA unifatorial e teste de Tukey para os descritores abióticos registrados próximos a piscicultura no Rio São Mateus.

\begin{tabular}{cccc}
\hline & & PONTOS & COLETAS \\
\hline \multirow{2}{*}{ \% Mat. Orgânica } & $\mathrm{F}$ & 2,38 & 0,52 \\
& $p$ & $\mathrm{~ns}$ & $\mathrm{~ns}$ \\
Seleção & $\mathrm{F}$ & 3,7 & 0,94 \\
& $p$ & $0,03(\mathrm{~J}>\mathrm{M})$ & $\mathrm{ns}$ \\
Média & $\mathrm{F}$ & 2,69 & 0,76 \\
& $p$ & $\mathrm{~ns}$ & $\mathrm{~ns}$ \\
\% Finos & $\mathrm{F}$ & 6,4 & 1,09 \\
\% Areia & $p$ & $0,005(\mathrm{OJ}>\mathrm{C})$ & $\mathrm{ns}$ \\
\multirow{2}{*}{ \% Cascalho } & $\mathrm{F}$ & 6,41 & 0,88 \\
& $p$ & $0,005(\mathrm{C}>\mathrm{OJ})$ & $\mathrm{ns}$ \\
& $\mathrm{F}$ & 2,01 & 1,39 \\
\hline
\end{tabular}

$p<0,05 . \mathrm{ns}=$ não significante. $\mathrm{J}=$ Jusante; $\mathrm{M}=$ Montante; $\mathrm{C}=$ Cultivo; $\mathrm{O}=$ Oposta. Fonte: Autores.

\section{Macrofauna}

Foram encontrados 195 organismos distribuídos em 5 táxons, com abundância média variando entre 0 e 24,3 indivíduos por ponto entre os meses amostrados (Tabela 5). Os oligoquetas Tubificidae sp1 obtiveram 53\% dos indivíduos registrados, seguido pelas larvas de Chironomidae Clinotanypus sp. (23\%) e Aedokritus sp. (15\%).

Tabela 5. Abundância média ( \pm DP) e riqueza taxonômica da macrofauna bentônica encontradas nas cinco amostragens realizadas próximas a uma área de cultivo de peixes em tanques-rede no rio São Mateus, estado do Espírito Santo, Brasil.

\begin{tabular}{|c|c|c|c|c|c|c|c|c|c|c|c|c|c|c|c|c|c|c|c|c|}
\hline & \multicolumn{4}{|c|}{ fev/11 } & \multicolumn{4}{|c|}{ ago/11 } & \multicolumn{4}{|c|}{ dez/11 } & \multicolumn{4}{|c|}{ abr/12 } & \multicolumn{4}{|c|}{ mai/12 } \\
\hline & $\mathrm{C}$ & $\mathbf{J}$ & $\mathbf{M}$ & 0 & $\mathrm{C}$ & $\mathbf{J}$ & $\mathbf{M}$ & 0 & $\mathrm{C}$ & $\mathbf{J}$ & $\mathbf{M}$ & 0 & $\mathrm{C}$ & $\mathbf{J}$ & $\mathbf{M}$ & 0 & $\mathrm{C}$ & $\mathbf{J}$ & $\mathbf{M}$ & 0 \\
\hline \multicolumn{21}{|l|}{ CHIRONOMIDAE } \\
\hline Aedokritus sp. & 0 & 0 & 0 & 1 & 0 & 0 & 0 & 0 & 0 & 1 & 1 & 0 & - & 2 & 6 & 1 & 13 & 3 & 2 & 0 \\
\hline Clinotanypus sp. & 1 & 0 & 0 & 0 & 0 & 0 & 0 & 0 & 0 & 2 & 0 & 0 & - & 4 & 0 & 5 & 3 & 3 & 24 & 3 \\
\hline \multicolumn{21}{|l|}{ TANAIDACEA } \\
\hline Tanaidaceo sp1 & 0 & 0 & 0 & 0 & 0 & 0 & 0 & 0 & 0 & 0 & 0 & 0 & - & 11 & 0 & 0 & 0 & 1 & 0 & 0 \\
\hline \multicolumn{21}{|l|}{ OLIGOCHAETA } \\
\hline Tubificidae sp1 & 2 & 0 & 0 & 0 & 3 & 0 & 0 & 0 & 5 & 9 & 6 & 1 & - & 0 & 4 & 1 & 19 & 1 & 47 & 5 \\
\hline Tubificidae sp2 & 0 & 1 & 0 & 0 & 4 & 0 & 0 & 0 & 0 & 0 & 0 & 0 & - & 0 & 0 & 0 & 0 & 0 & 0 & 0 \\
\hline $\begin{array}{l}\text { Média } \\
\text { (DP) }\end{array}$ & $\begin{array}{c}1 \\
(1,7)\end{array}$ & $\begin{array}{c}0,3 \\
(0,6)\end{array}$ & 0 & $\begin{array}{c}0,3 \\
(0,6)\end{array}$ & $\begin{array}{l}2,3 \\
(4)\end{array}$ & $\mathbf{0}$ & 0 & 0 & $\begin{array}{c}1,7 \\
(1,5)\end{array}$ & $\begin{array}{c}4 \\
(5,2)\end{array}$ & $\begin{array}{c}2,3 \\
(2,1)\end{array}$ & $\begin{array}{c}0,3 \\
(0,6)\end{array}$ & - & $\begin{array}{c}5,7 \\
(4,7)\end{array}$ & \begin{tabular}{|c|}
3,3 \\
$(3,2)$
\end{tabular} & $\begin{array}{c}2,3 \\
(0,6)\end{array}$ & $\begin{array}{l}11,7 \\
(3,5)\end{array}$ & $\begin{array}{c}2,7 \\
(1,5)\end{array}$ & $\begin{array}{l}24,3 \\
(17)\end{array}$ & $\begin{array}{c}2,7 \\
(1,2)\end{array}$ \\
\hline Riqueza & 2 & 1 & $\mathbf{0}$ & 1 & 2 & 0 & 0 & 0 & 1 & 3 & 2 & 1 & - & 3 & 2 & 3 & 3 & 4 & 3 & 2 \\
\hline
\end{tabular}

\section{๔®๑}


Citação (APA): Rezeno, J. L., Costa, K. G., \& Teodoro, N. M. S. (2020). Macrofauna bentônica sob efeito da piscicultura de tanque-rede no rio São Mateus, sudeste do Brasil. Brazilian Journal of Production Engineering, 6(8), Edição Especial "Pesca e Aquicultura: Gestão, Política e Inovação", 01-13.

Foi nítido o aumento dos valores médios da abundância e riqueza dos organismos ao longo do tempo, com os meses de abril e maio/12 registrando valores significativamente maiores que os demais meses $(p<0,05)$. Os valores de abril/12, para ambos os parâmetros, foram significativamente maiores que fevereiro/11 e agosto/11, enquanto que os valores registrados em maio/12 foram significativamente maiores que os meses de fevereiro/11, agosto/12 e dezembro/11 (Tabela 6). Em relação às diferenças entre os pontos amostrados, não houveram resultados significativos dentro e entre as campanhas. Quanto a diversidade de ShannonWiener, apenas o valor registrado em maio/12 foi significativamente maior que os demais meses ( $p<0,05$, Tabela 6 ). Novamente não houve diferença significativa entre os pontos dentro e entre as campanhas.

Tabela 6. Resultado da ANOVA bifatorial para os índices univariados da macrofauna bentônica encontradas em cinco amostragens realizadas próximas a uma área de cultivo de peixes em tanques-rede no rio São Mateus, estado do Espírito Santo, Brasil.

\begin{tabular}{cccc}
\hline VARIÁVEIS & & $\mathbf{F}$ & $\boldsymbol{P}(<\mathbf{0 , 0 5})$ \\
\hline \multirow{2}{*}{ ABUNDÂNCIA } & pontos & 2,19 & $\mathrm{~ns}$ \\
& meses & 22,34 & $<0,001$ \\
& pontos x meses & 1,73 & $\mathrm{~ns}$ \\
RIQUEZA & pontos & 0,15 & $\mathrm{~ns}$ \\
& meses & 20,57 & $<0,001$ \\
& pontos x meses & 0,94 & $\mathrm{~ns}$ \\
DIVERSIDADE & pontos & 0,65 & $\mathrm{~ns}$ \\
& meses & 11,94 & $<0,001$ \\
& pontos x meses & 0,8 & $\mathrm{~ns}$ \\
\hline
\end{tabular}

Fonte: Autores.

As análises multivariadas MDS e ANOSIM também detectaram diferenças na estrutura da macrofauna apenas entre os meses de coletas. Na figura 2A, nota-se que os pontos se encontram misturados e dispersos, não sendo possível visualizar um agrupamento entre as amostras do mesmo ponto amostral. No entanto, na figura 2B foi possível visualizar que as amostras dos meses de abril e maio/12 estão aparentemente mais agrupadas que as demais. O ANOSIM confirma que somente há diferenças significativas entre os meses (R Global: 0,246; $p<0,05$ ). A maioria das combinações par-a-par entre meses apresentou diferença significativa, e apenas as combinações fev/11 e ago/11; dez/11 e abr/12; e abr/12 e mai/12 se mostraram semelhantes entre si. 
Citação (APA): Rezeno, J. L., Costa, K. G., \& Teodoro, N. M. S. (2020). Macrofauna bentônica sob efeito da piscicultura de tanque-rede no rio São Mateus, sudeste do Brasil. Brazilian Journal of Production Engineering, 6(8), Edição Especial "Pesca e Aquicultura: Gestão, Política e Inovação", 01-13.

Figura 2. Análise de ordenação (MDS) da macrofauna bentônica encontradas nas cinco amostragens (fev/11, ago/11, dez/11, abr/12 e mai/12) do estudo. A) Entre os pontos de amostragens; B) entre os meses de coletas.

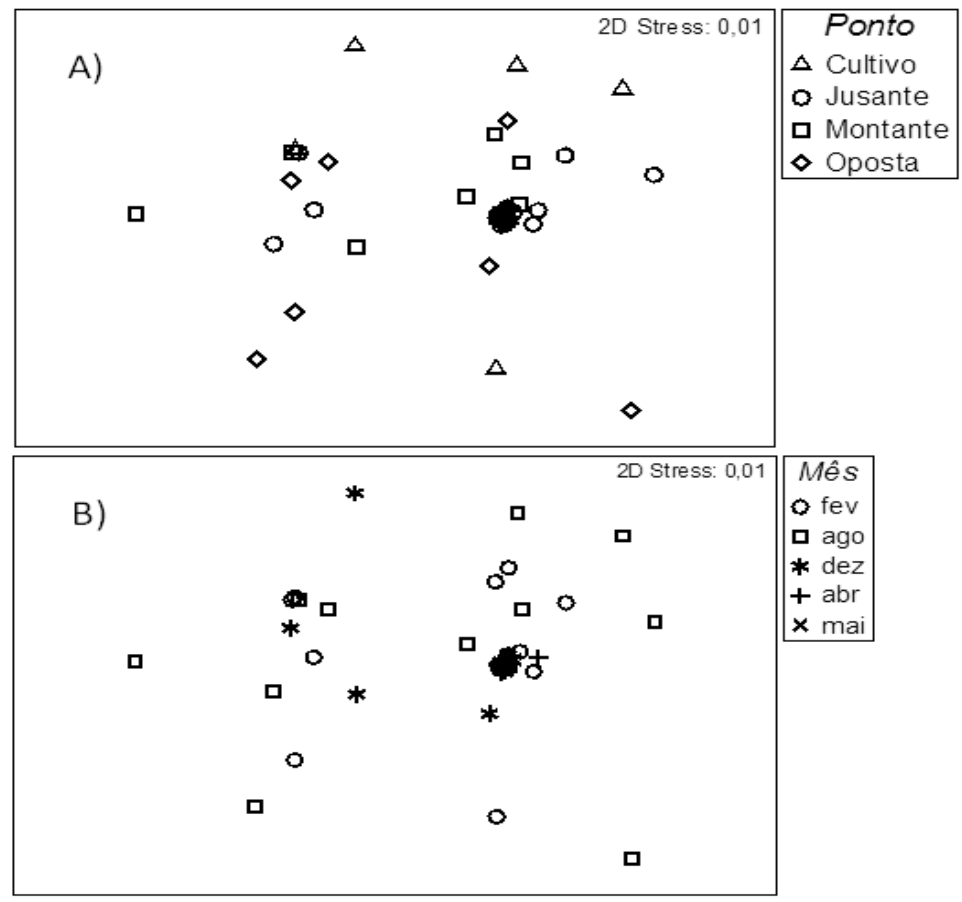

Fonte: Autores.

De acordo com o índice biológico BMWP', a somatória das pontuações atribuídas às famílias dos indivíduos coletados classificou a qualidade da água como "muito crítica" em todos os pontos estudados ao longo dos meses (Tabela 7).

Tabela 7. Soma dos valores do índice BMWP' atribuídos aos organismos por coleta e a interpretação da qualidade da água segundo Alba-Tercedor (1996).

\begin{tabular}{|c|c|c|c|c|c|c|c|c|c|c|c|c|c|c|c|c|c|c|c|c|}
\hline & \multicolumn{4}{|c|}{ fev/11 } & \multicolumn{4}{|c|}{ ago/11 } & \multicolumn{4}{|c|}{ dez 11} & \multicolumn{4}{|c|}{$\mathrm{abr} / 12$} & \multicolumn{4}{|c|}{$\mathrm{mai} / 12$} \\
\hline & C & $\mathrm{J}$ & M & 0 & C & $\mathbf{J}$ & M & 0 & C & $\mathrm{J}$ & M & 0 & C & $\mathrm{J}$ & M & 0 & C & $\mathrm{J}$ & M & 0 \\
\hline $\begin{array}{l}\text { Pontuaçao } \\
\text { BMWP' }\end{array}$ & 3 & 1 & & 2 & 1 & & & & 1 & 3 & 3 & 1 & & 3 & 3 & 3 & 3 & 3 & 3 & 3 \\
\hline Ualida & & & & & & & & & & & & & & & & & & & & \\
\hline da água & $\mathrm{mc}$ & $\mathrm{mc}$ & & $\mathrm{mc}$ & $\mathrm{mc}$ & & & & $\mathrm{mc}$ & $\mathrm{mc}$ & $\mathrm{mc}$ & $\mathrm{mc}$ & & $\mathrm{mc}$ & $\mathrm{mc}$ & $\mathrm{mc}$ & $\mathrm{mc}$ & $\mathrm{mc}$ & $\mathrm{mc}$ & $\mathrm{mc}$ \\
\hline
\end{tabular}

Os espaços em branco na tabela correspondem aos pontos onde não foram encontrados organismos. $\mathrm{mc}=$ muito crítica $\mathrm{C}=$ cultivo; $\mathrm{J}=$ jusante; $\mathrm{M}=$ montante $\mathrm{O}=$ oposta.

Fonte: Autores.

Alterações na estrutura da comunidade bentônica foram pouco relacionadas com variáveis ambientais (BIO-ENV). O melhor valor de $\rho$ para a macrofauna foi de 0,060 (matéria orgânica), considerado baixo para essa analise.

\section{DISCUSSÃO}

A hipótese do presente trabalho, que houvesse diferença na estrutura da comunidade macrobentônica abaixo do cultivo de peixes em relação aos outros pontos amostrais foi refutada, visto que as análises uni- e multivariadas não identificaram diferenças entre os locais.

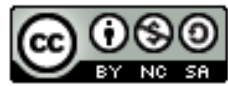


Citação (APA): Rezeno, J. L., Costa, K. G., \& Teodoro, N. M. S. (2020). Macrofauna bentônica sob efeito da piscicultura de tanque-rede no rio São Mateus, sudeste do Brasil. Brazilian Journal of Production Engineering, 6(8), Edição Especial "Pesca e Aquicultura: Gestão, Política e Inovação", 01-13.

Segundo Lin \& Yi (2003), na piscicultura intensiva há considerável aumento de alimento e resíduos, inclusive material orgânico. Ainda, de acordo com Pearson \& Gowen (1990) e Silva, Silva, Araújo \& Faustino (2013) cerca de $20 \%$ da ração utilizada para alimentação dos peixes estocados em tanques-rede é perdida, antes de ser ingerida. No entanto, a matéria orgânica não diferiu entre os pontos amostrados.

De acordo com Rotta \& Queiroz (2003), o acúmulo de ração ou dos seus resíduos nos sedimentos, provenientes da piscicultura em tanques-redes, poderia aumentar o carreamento de sedimentos para outras áreas, devido às correntes de água existentes. Além disso, o despejo de esgoto doméstico que ocorre próximo ao cultivo também pode ter contribuído para os baixos valores dos parâmetros da macrofauna registrados na região, afetando até mais que o cultivo. Ainda segundo Rotta \& Queiroz (2003), a existência de resíduos de agroquímicos provenientes do aporte de matéria orgânica e resíduos urbanos das cidades devem ser levados em consideração, para que as alterações ambientais negativas provocadas por fatores externos não venham a ser erroneamente atribuídas somente à piscicultura.

No caso do Rio São Mateus, o esgoto doméstico já foi apontado por Assis \& Freitas, 2012 e além de toda uma degradação histórica, causada pelo alto nível de antropização, principalmente por atividades como pastagens, cultivos agrícolas e silvicultura que interferem em todo o recurso hídrico, incluindo a qualidade dos sedimentos depositados no fundo (AGERH, 2018). No trabalho de Gomes, Rusciolelli, Silva, Nascimento, Dadalto, \& Freitas (2015) foram feitas entrevistas com os pescadores da região de Pedra d'Água que relataram que a quantidade de pescados tem diminuído nos últimos anos. Em estudos realizados em 2010 e 2011, que avaliaram aspectos limnológicos do rio, inclusive no trecho onde foi realizado o presente estudo, os autores concluíram que as áreas onde estão localizadas a piscicultura e o lançamento de efluentes domésticos apresentaram as piores condições ambientais do rio (Mamão, 2012; Pereira, 2012; Almeida, 2012).

O índice BMWP' indicou que a qualidade ambiental da área onde estão implantados os tanquesredes é muito crítica, uma vez que apresentou valores ruins quanto à fauna encontrada, constituída basicamente por larvas de Chironomidae e oligoquetas, organismos que com sua ampla diversidade de hábitos e funções alimentares, atingiram adaptabilidade fisiológica para viver em condições extremas (Pastorino, Pizzul, Bertoli, Perilli, Brizio, \& Salvi, 2019).

O grande aporte de matéria orgânica encontrado no sedimento da área de estudo, proveniente de distintas fontes poluidoras, incluindo a própria piscicultura e o lançamento de efluentes domésticos da região, possivelmente está interferindo negativamente na qualidade do sedimento do fundo, o que justifica a abundância desses organismos. Ainda, as variações temporais registradas nos parâmetros da comunidade bentônica, com aumento gradativo dos valores de abundância, riqueza e diversidade de organismos ao longo do tempo, demostram que possíveis alterações, naturais ou antrópicas, nas condições ambientais do rio podem estar interferindo na qualidade da fauna bentônica e diluindo um possível efeito causado pelo cultivo.

Assim como observado no presente estudo, Alongi, Chong, Dixon, Sasekumar, \& Tirendi (2003) também tiveram dificuldades em detectar impactos causados por tanques-redes em áreas

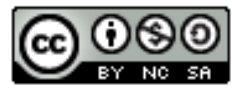


Citação (APA): Rezeno, J. L., Costa, K. G., \& Teodoro, N. M. S. (2020). Macrofauna bentônica sob efeito da piscicultura de tanque-rede no rio São Mateus, sudeste do Brasil. Brazilian Journal of Production Engineering, 6(8), Edição Especial "Pesca e Aquicultura: Gestão, Política e Inovação", 01-13.

estuarinas que recebem massivas quantidades de matéria orgânica na Malásia. Além disso, as características do efluente gerado pela piscicultura são bastante semelhantes àquelas do efluente doméstico, apresentando, por exemplo, elevada demanda bioquímica de oxigênio e grande concentração de sólidos em suspensão, compostos nitrogenados e fosfatados (Avnimelech, 1999). Em um trabalho sobre criação de Oncorhynchus mykiss em Quebec (Cornel, \& Whoryskey, 1993), o sedimento localizado abaixo de tanques-rede apresentava porcentagem de matéria orgânica semelhante à de áreas que recebem aporte de material alóctone.

\section{CONCLUSÃO}

A degradação ambiental causada por diversos fatores, incluindo a descarga de efluentes orgânicos, na área de implantação dos tanques-redes no estuário do rio São Mateus parece comprometer muito mais a fauna bentônica do local do que a própria piscicultura. $\mathrm{O}$ índice BMWP' classificou como muito crítica a qualidade ambiental desse trecho. Dessa forma, esperamos que as informações fornecidas por esse trabalho, juntamente com mais estudos sobre a qualidade de água e do sedimento do rio, o mapeamento de pontos ilegais ou não regulamentados de descarga de efluentes, tratamento de esgoto, recuperação de margens de rios e o estabelecimento de práticas agrícolas sustentáveis, possam ser utilizadas para auxiliar a elaboração de ações visando a melhoria nas condições ambientais do rio São Mateus e, consequentemente, na qualidade de vida da comunidade do entorno.

\section{AGRADECIMENTOS}

APESAM - Associação de Pescadores de São Mateus, Dr. Rodrigo Randow de Freitas pelo apoio logístico durante as coletas e a equipe do Núcleo de Bentos Marinho (NUBEM/UFES). Este trabalho foi realizado como Trabalho de Conclusão de Curso em Ciências Biológicas de J.L.R. e foi um subprojeto da Rede Capixaba de Pesquisa com Robalo-Peva (Centropomus parallelus), Fase II: Consolidação (RECAPER). O projeto teve apoio financeiro do CNPq.

\section{REFERÊNCIAS}

AGERH - Agência Estadual de Recursos Hídricos. (2018). Recuperado em 24 junho, 2020, de https://agerh.es.gov.br/Media/agerh/Documenta\%C3\%A7\%C3\%A3o\%20CBHs/S\%C3\%A3o $\%$ 20Mateus/Plano\%20de\%20Bacia/REA_Diagn\%C3\%B3stico\%20Progn\%C3\%B3stico_CB H\%20S\%C3\%A3oMateus.pdf.

Alba-Tercedor, J. (1996). Macroinvertebrados acuáticos y calidad de las aguas de los ríos. IV SIAGA, Almeria, 2: 203-213.

Almeida, L. B. (2012). Estrutura e dinâmica da comunidade fitoplanctônica em um trecho do rio são Mateus (São Mateus - ES) com piscicultura intensiva (tanques-rede). Trabalho de conclusão de curso, Universidade Federal do Espírito Santo, São Mateus, Espírito Santo, Brasil.

Alongi, D. M., Chong, V.C., Dixon, P., Sasekumar, A., \& Tirendi, F. (2003). The influence of fish cage aquaculture on pelagic carbon flow and water chemistry in tidally dominated mangrove estuaries of peninsular Malaysia. Marine Environmental Research, 55(4): 313-333. doi: 10.1016/s0141-1136(02)00276-3.

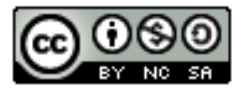


Citação (APA): Rezeno, J. L., Costa, K. G., \& Teodoro, N. M. S. (2020). Macrofauna bentônica sob efeito da piscicultura de tanque-rede no rio São Mateus, sudeste do Brasil. Brazilian Journal of Production Engineering, 6(8), Edição Especial "Pesca e Aquicultura: Gestão, Política e Inovação", 01-13.

Assis, M.C. \& Freitas, R.R. (2012). Análise das práticas de biossegurança no cultivo de tilápias (Oreochromis niloticus) em região estuarina no sudeste do Brasil. Revista da Gestão Costeira Integrada, 12(4):559-568.

Avnimelech, Y. (1999). Carbon/nitrogen ratio as a control element in aquaculture systems. Aquaculture, 176: 227 - 235. doi: 10.1016/S0044-8486(99)00085-X.

Barbosa, A. C. A., Moura, E. V. M., \& Santos, R. V. (2010). Cultivo de tilápias em gaiolas. Empresa de Pesquisa Agropecuária do Rio Grande do Norte, Natal, Rio Grande do Norte, 33p. $\begin{array}{lllll}\text { Recuperado } & \text { em } & 1 & \text { julho, } & 2020\end{array}$ http://files.jornalagroinformativo.webnode.com.br/200000020415f942597/cartilha_cultivo_de_tilapias_em_gaiolas.pdf.

Barroso, R. M., Muñoz, A. E. P., \& Cai, J. (2019). Social and economic performance of tilapia farming in Brazil. FAO Fisheries and Aquaculture Circular, 1181: 1181. Recuperado em 30 junho, 2020 de http://www.fao.org/3/ca5304en/ca5304en.pdf.

Cardoso, A. S., El-Deir, S. G., \& Cunha, M. C. C. (2016). Bases da sustentabilidade para atividade de piscicultura no semi-árido de Pernambuco. Interações, 17 (4): 645 - 653. doi: dx.doi.org/10.20435/1984-042X-2016-v.17-n.4(08)

Ceneviva-Bastos, M., Prates, D. B., Romero, R. M., Bispo, P. C., Casatti, L. (2017). Trophic guilds of EPT (Ephemeroptera, Plecoptera, and Trichoptera) in three basins of the Brazilian Savanna. Limnologica. 63: 11-17. doi: dx.doi.org/10.1016/j.limno.2016.12.004.

Clarke, K. R., \& Green, R. H. (1988). Statistical design and analysis for a 'biological effects' study. Marine Ecology Progress Series, 46: 213-226. Recuperado em 30 junho, 2020 de https://www.int-res.com/articles/meps/46/m046p213.pdf.

Clarke, K. R., \& Ainsworth, M. (1993). A method of linking multivariate community structure to environmental variables. Marine Ecology Progress Series, 92, 205-219.

Cornel, G. E. \& Whoriskey, F. G. (1993). The effects of rainbow trout (Oncorhynchus mykiss) cage culture on the water quality, zooplâncton, benthos and sediment of Lac du Passage, Quebec. Aquaculture, 109: 101-117. doi: 10.1016/0044-8486(93)90208-G.

Gomes, V. A. P., Rusciolelli, V. B., Silva, I. R. P., Nascimento, J. C., Dadalto, M. C. \& Freitas, R. R. (2015). História oral de pescadores da comunidade de Pedra d'Água, São Mateus, ES. Brazilian Journal of Production Engineering, 1(1): 1-3. doi: 10.30712/guara.v1i6.15123.

Griffiths, J.R., Kadin, M., Nascimento, F.J.A., Tamelander, T., Törnroos, A., Bonaglia, S., Bonsdorff, E., Brüchert, V., Gardmark, A., Järnströn, Kotta, J., Lindegren, M., Nordstrom, M., Norkko, A., Olsson, J., Weigel, B., Zydelis, R., Blenckner, T., Niiranen, S., Winder, M. (2017). The importance of benthic-pelagic coupling for marine ecosystem functioning in a changing world. Global Change Biology, 23, 2179-2196, doi: 10.1111/gcb.13642.

Huang, Y. C. A., Huang, S. C., Hsieh, H.J., Meng, P. J., \& Chen, C. A. (2012). Changes in sedimentation, sediment characteristics, and benthic macrofaunal assemblages around marine cage culture under seasonal monsoon scales in a shallow-water bay in Taiwan. Journal of Experimental Marine Biology and Ecology, 422-423: 55-63. doi: 10.1016/j.jembe.2012.04.008.

Lin, C. K., \& Yi, Y. (2003) Minimizing environmental impacts of freshwater aquaculture and reuse of pond effluents and mud. Aquaculture, 226: 57-68. doi: 10.1016/S00448486(03)00467-8.

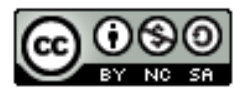


Citação (APA): Rezeno, J. L., Costa, K. G., \& Teodoro, N. M. S. (2020). Macrofauna bentônica sob efeito da piscicultura de tanque-rede no rio São Mateus, sudeste do Brasil. Brazilian Journal of Production Engineering, 6(8), Edição Especial "Pesca e Aquicultura: Gestão, Política e Inovação", 01-13.

Mamão, M.A. (2012). Ecologia do fitoplâncton de um rio tropical (rio São Mateus, ES). Dissertação de Mestrado, Universidade Federal do Espírito Santo, São Mateus, Espírito

Martins, A. C. (2016). Como montar um negócio para criação de peixes. Recuperado em 25 junho, 2020 , de https://m.sebrae.com.br/Sebrae/Portal\%20Sebrae/UFs/RN/Anexos/Aquicultura\%20\%20Como $\% 20$ montar\%20um\%20neg\%C3\%B3cio\%20para\%20cria\%C3\%A7\%C3\%A3o\%20de\%20pei xes.pdf

Menezes, L. C. B., \& Beyruth, Z. (2003). Impactos da aquicultura em tanques-rede sobre a comunidade bentônica da represa de Guarapiranga - São Paulo- SP. Boletim do Instituto de Pesca, 29(1): $77 \quad-86 . \quad$ Recuperado em 1 julho, 2020 de https://www.pesca.sp.gov.br/Menezes.PDF.

Moura, R. S. T., Lopes, Y. V. A. \& Henry-Silva, G. G. (2014). Sedimentação de nutrientes e material particulado em reservatório sob influência de atividades de piscicultura no semiárido do Rio Grande do Norte. Química Nova, 37 (8): 1283 - 1288. doi: dx.doi.org/10.5935/01004042.20140203.

Óscar, P. M., Lindsay, R., Trevor, T., \& Barquin, L. M. (2003). Water quality requirements for marine fish cage site selection in Tenerife (Canary Islands): predictive modelling and analysis using GIS. Aquaculture, 224:51-68. doi: 10.1016/S0044-8486(02)00274-0.

Paiva, R. P., Casotti, R., Rodrigues, R. L., \& Freitas, R. R. (2015). Cultivo e beneficiamento de Oreochromis niloticus e Centropomus parallelus em região estuarina no sudeste do Brasil. Acta of Fisheries and Aquatic Resources, 3(1): 54-64. doi: 10.2312/ActaFish.2015.3.1.54-64.

Pastorino, P., Pizzul, E., Bertoli, M., Perilli, S., Brizio, P., \& Salvi, G. (2019). Macrobenthic invertebrates as bioindicators of trace elements in high-mountain lakes. Environmental Science and Pollution Research. 27(6):5958-5970. doi: 10.1007/s11356-019-07325-x.

Pereira, T. A. (2012). Distribuição longitudinal da comunidade perifítica no rio São Mateus (norte do Espírito Santo). Dissertação de mestrado, Universidade Federal do Espírito Santo, São Mateus, Espírito Santo, Brasil.

PROATER- Programa de Assistência Técnica e Extensão Rural (2011). Disponível em: https://incaper.es.gov.br/media/incaper/proater/municipios/Nordeste/Sao_Mateus.pdf.

Rezeno, J. L. (2012) - Impactos da piscicultura sobre a macrofauna bentônica no rio São Mateus, ES. Monografia, Universidade Federal do Espírito Santo, São Mateus, ES, Brasil.

Rotta, M. A., \& Queiroz, J. F. (2003). Boas práticas de manejo (BPMs) para a produção de peixes em tanques-rede. Recuperado em 26 junho, 2020, de https://ainfo.cnptia.embrapa.br/digital/bitstream/item/81186/1/DOC47.pdf

Silva, R.F., Silva, J.L.A., Araújo, M.S.B., \& Faustino, O, W. C. (2013). Qualidade de resíduo de tanques de produção de alevinos como condicionante de solos do semiárido de Pernambuco: subsídios para gestão ambiental. Gaia Scientia, 7(1): 58-63.

Suguio, K. (1973). Introdução à sedimentologia. São Paulo, Brasil. Edgard Blücher/EDUSP.

Vieira, J. L. M. (2018). Nutrição do tambaqui (Colossoma macropomum, Cuvier, 1818) na região norte do Brasil. Desarrolo Local Sostenible.

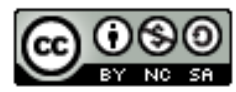

